\title{
Quasisymmetric Schur functions
}

\author{
James Haglund $1 \|$ Sarah Mason ${ }^{2}$, Kurt Luoto ${ }^{3}$ and Steph van Willigenburg 蚆 \\ ${ }^{1}$ University of Pennsylvania, Philadelphia, PA 19104-6395, USA \\ ${ }^{2}$ Davidson College, Davidson, NC 28035-7129, USA \\ ${ }^{3}$ University of Washington, Seattle, WA 98195-4350, USA \\ ${ }^{4}$ University of British Columbia, Vancouver, BC V6T 1Z2, CANADA
}

\begin{abstract}
We introduce a new basis for the algebra of quasisymmetric functions that naturally partitions Schur functions, called quasisymmetric Schur functions. We describe their expansion in terms of fundamental quasisymmetric functions and determine when a quasisymmetric Schur function is equal to a fundamental quasisymmetric function. We conclude by describing a Pieri rule for quasisymmetric Schur functions that naturally generalizes the Pieri rule for Schur functions.

Résumé. Nous étudions une nouvelle base des fonctions quasisymetrique, les fonctions de quasiSchur. Ces fonctions sont obtenues en spécialisant les fonctions de Macdonald dissymétrique. Nous décrivons les compositions que donnes un simple fonction quasisymetrique. Nous décrivons aussi une règle par certaines fonctions de Schur.
\end{abstract}

Keywords: nonsymmetric Macdonald polynomials, Pieri rule, quasisymmetric functions, Schur functions

\section{Introduction}

The Schur functions form an important basis for all symmetric functions, indexed by integer partitions. They appear in the theory of Macdonald polynomials, (4), which are $q, t$-analogues of symmetric functions that were introduced to study problems from algebraic geometry. More precisely, the Schur function $s_{\lambda}$ is the specialization to $q=t=0$ of the Macdonald polynomial, $P_{\lambda}(X ; 0,0)$.

The so-called nonsymmetric Macdonald polynomials, denoted $E_{\alpha}(X ; q, t)$ where $\alpha$ is a composition, are a basis for the polynomial ring which share many of the $P_{\lambda}$ 's nice properties, such as orthogonality. The coefficients in the expansion of $P_{\lambda}(X ; q, t)$ in terms of the $E_{\alpha}(X ; 1 / q, 1 / t)$ appear in Macdonald's work (5) (see also (3) Prop. 5.3.1)), and setting $q=t=0$ in this identity implies that the Schur functions are decomposed into nonsymmetric functions

$$
E_{\gamma_{n}, \ldots, \gamma_{1}}\left(x_{n}, \ldots, x_{1} ; \infty, \infty\right)=N S_{\gamma}(X)
$$

that are indexed by compositions. These functions, often referred to as Demazure atoms since they are characters of quotients of Demazure modules, exhibit many of the properties of Schur functions, including

\footnotetext{
${ }^{\dagger}$ Supported in part by NSF grant DMS 0553619.

$\ddagger$ Supported in part by the National Sciences and Engineering Research Council of Canada. 
an analogue of the Robinson-Schensted-Knuth Algorithm (6). Since the quasisymmetric functions, (2), form a bridge between nonsymmetric and symmetric functions, it is natural to seek a quasisymmetric function that bridges the gap between nonsymmetric Schur functions and Schur functions.

This paper presents a class of quasisymmetric functions that arises from the nonsymmetric Schur functions and decomposes the Schur functions. These functions, which we call quasisymmetric Schur functions, form a basis for the algebra of quasisymmetric functions. In Section 2 we define these functions and describe their connection to the basis of fundamental quasisymmetric functions. In Section 3 we describe a Pieri rule for multiplication of a quasiymmetric Schur function by a quasisymmetric Schur function indexed by a simple composition.

\subsection{Quasisymmetric and symmetric functions}

A quasisymmetric function is a bounded degree formal power series in variables $X=\left\{x_{1}, x_{2}, \ldots\right\}$ such that for all $k$ and $i_{1}<i_{2}<\cdots<i_{k}$ the coefficient of $x_{i_{1}}^{\alpha_{1}} x_{i_{2}}^{\alpha_{2}} \ldots x_{i_{k}}^{\alpha_{k}}$ is equal to the coefficient of $x_{1}^{\alpha_{1}} x_{2}^{\alpha_{2}} \ldots x_{k}^{\alpha_{k}}$. The set of all quasisymmetric functions forms a graded algebra $\mathcal{Q}=\mathcal{Q}_{0} \oplus \mathcal{Q}_{1} \ldots$.

Two natural bases for quasisymmetric functions are the monomial basis $\left\{M_{\alpha}\right\}$ and the fundamental basis $\left\{F_{\alpha}\right\}$ indexed by compositions $\alpha=\left(\alpha_{1}, \alpha_{2}, \ldots, \alpha_{k}\right)$, where $\alpha_{i}>0$ for all $i$. The monomial basis is spanned by $M_{0}=1$ and all power series

$$
M_{\alpha}=\sum_{i_{1}<i_{2}<\cdots<i_{k}} x_{i_{1}}^{\alpha_{1}} x_{i_{2}}^{\alpha_{2}} \cdots x_{i_{k}}^{\alpha_{k}}
$$

The fundamental basis is spanned by $F_{0}=1$ and all power series

$$
F_{\alpha}=\sum_{\gamma \leq \alpha} M_{\gamma}
$$

where $\gamma \leq \alpha$ if $\alpha$ can be obtained from $\gamma$ by adding together adjacent parts of $\gamma$. In this case, $\alpha$ is called a coarsening of $\gamma$. For example, $(3,5,2,3,2) \geq(3,2,1,2,1,1,3,2)$.

Furthermore, $\mathcal{Q}_{n}=\operatorname{span}_{\mathbb{Q}}\left\{\mathrm{M}_{\alpha} \mid \alpha \vDash \mathrm{n}\right\}=\operatorname{span}_{\mathbb{Q}}\left\{\mathrm{F}_{\alpha} \mid \alpha \vDash \mathrm{n}\right\}$, where we write $\alpha \vDash n$ to indicate that $\alpha$ is a composition of $n$. We define the algebra of symmetric functions $\Lambda$ as the subalgebra of $\mathcal{Q}$ spanned by the monomial symmeric functions

$$
m_{\lambda}=\sum_{\alpha: \lambda(\alpha)=\lambda} M_{\alpha}, \quad \lambda \vdash n, n>0
$$

where $\lambda(\alpha)$ is the partition obtained by arranging the parts of $\alpha$ in weakly decreasing order and $\lambda \vdash n$ means that $\lambda$ is a partition of $n$. The algebra $\Lambda=\Lambda_{0} \oplus \Lambda_{1} \cdots$ is a graded algebra, with $\Lambda_{n}=\Lambda \cap \mathcal{Q}_{n}$.

Perhaps the most well-known basis for $\Lambda$ is the basis of Schur functions, $\left\{s_{\lambda}\right\}$, which we now define combinatorially. If $\lambda \vdash n$, then its associated Ferrers diagram is the array of left justified boxes, or cells, with $\lambda_{i}$ cells in the $i$-th row from the top. We abuse notation by using $\lambda$ to refer to both the partition $\lambda$ and its Ferrers diagram. A semi-standard Young tableau (SSYT) of shape $\lambda$ is a map from the cells of $\lambda$ to $\mathbb{Z}^{+}$(or a filling of the cells of $\lambda$ ) such that the row entries are weakly increasing from left to right, and the column entries are strictly increasing from top to bottom. For example, 


$$
T=\begin{array}{|l|l|l|l|}
\hline 1 & 2 & 2 & 4 \\
\hline 2 & 3 & 3 & \\
\cline { 1 - 2 } 3 & 4 & 4 & \\
\cline { 1 - 2 } 4 & 6 & \multicolumn{2}{|}{} \\
\cline { 1 - 2 } 6 & \multicolumn{2}{|c}{} \\
\cline { 1 - 1 }
\end{array}
$$

Each SSYT $T$ has an associated weight $x^{T}=x_{1}^{a_{1}} x_{2}^{a_{2}} \cdots$, where $a_{i}$ is the number of times the positive integer $i$ appears in $T$. The weight of $T$ in the above example is $x^{T}=x_{1} x_{2}^{3} x_{3}^{3} x_{4}^{4} x_{6}^{2}$.

The Schur function $s_{\lambda}$ is then defined by

$$
s_{\lambda}=\sum_{T} x^{T},
$$

where the sum is over all SSYTx of shape $\lambda$. The Schur functions can also be realized as a sum of fundamental quasisymmetric functions as follows. Given a standard Young tableau (SYT), that is, a SSYT with weight $\prod_{i=1}^{n} x_{i}$, its descent set $D(T)$ is the set of all $i$ such that $i+1$ appears in a lower row of $T$ than $i$. Then

$$
s_{\lambda}=\sum_{T} F_{\beta(D(T))},
$$

where the sum is over all SYTx $T$ of shape $\lambda$, and if $S=\left\{i_{1}, i_{2}, \ldots, i_{k-1}\right\} \subseteq[n-1]$, then $\beta(S)=$ $\left(i_{1}, i_{2}-i_{1}, i_{3}-i_{2}, \ldots, n-i_{k-1}\right)$.

\subsection{A specialization of nonsymmetric Macdonald polynomials}

By combining the specialization of the nonsymmetric Macdonald polynomials to Demazure atoms mentioned in the introduction with the combinatorial formula for nonsymmetric Macdonald polynomials provided by Haglund, Haiman, and Loehr (3), we obtain a description of Demazure atoms in terms of new combinatorial objects which we now describe.

Let $\gamma=\left(\gamma_{1}, \gamma_{2}, \ldots\right)$ be a weak composition of $n$. The augmented column diagram of $\gamma$ is a figure $\widehat{d g^{\prime}}(\gamma)$ consisting of $n$ cells arranged into columns as in (3) such that the $i^{\text {th }}$ column contains $\gamma_{i}+1$ cells. The bottom row of the diagram is called the basement, and its cells are filled with the integers $[n]$, where each integer from 1 to $n$ appears exactly once as shown below.

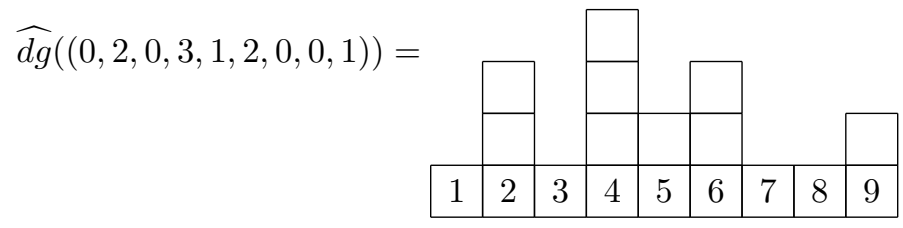

An augmented filling, $\sigma$, of an augmented diagram $\widehat{d g}(\gamma)$ is an assignment of positive integer entries to the cells of $\gamma$. We describe conditions on the entries in the cells that enable us to define an analogue of semi-standard Young tableaux. 
Three cells $\{a, b, c\} \in \widehat{d g}(\gamma)$ are called a type A triple if they are situated as follows

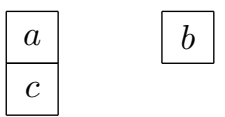

where $a$ and $b$ are in the same row, possibly the first row, possibly with cells between them, $c$ is directly below $a$, and the height of the column containing $a$ and $c$ is greater than or equal to the height of the column containing $b$. We say that the cells $a, b, c$ form a type $A$ inversion triple if their entries, ordered from smallest to largest, form a counter-clockwise orientation. If two entries are equal, then the entry which appears first when the entries are read left to right, top to bottom is considered smallest.

Similarly, three cells $\{a, b, c\} \in \lambda$ are a type $B$ triple if they are situated as shown,

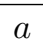

$$
\begin{array}{|l|}
\hline c \\
\hline b \\
\hline
\end{array}
$$

where $a$ and $b$ are in the same row (possibly the basement), possibly with cells between them, $c$ is directly on top of $b$, and the column containing $b$ and $c$ is strictly taller than the column containing $a$. We say that the cels $a, b, c$ form a type $B$ inversion triple if their entries, when ordered from smallest to largest, form a clockwise orientation. Again, when two entries are equal, the entry which appears first when the entries are read left to right, top to bottom is considered smallest.

Define a semi-skyline augmented filling (SSAF) of an augmented diagram $\widehat{d g}(\gamma)$ to be an augmented filling $F$ such that the entries in each column (read top to bottom) are weakly increasing and every triple is an inversion triple. Specializing the combinatorial formula for the nonsymmetric Macdonald polynomials $E_{\gamma}(X ; q, t)$ given in (3) and applying Corollary 2.4 of (6) implies that the Demazure atom, $N S_{\gamma}(X)$ is given by

$$
N S_{\gamma}(X):=E_{\gamma_{n}, \ldots, \gamma_{1}}\left(x_{n}, \ldots, x_{1} ; \infty, \infty\right)=\sum_{F \in S S A F(\widehat{d g}(\gamma))} x^{F},
$$

where $S S A F(\widehat{d g}(\gamma))$ is the set of all semi-skyline augmented fillings of shape $\gamma$.

\section{Quasisymmetric Schur functions}

Let $\gamma$ be a weak composition of $n$. Removing the zeros from $\gamma$ produces a (strong) composition, $\alpha$, of $n$, called the collapse of $\gamma$. We say that $\gamma$ collapses to $\alpha$ if $\alpha$ is the collapse of $\gamma$.

Definition 2.1 Let $\alpha$ be a composition of $n$. The quasisymmetric Schur function $\mathcal{S}_{\alpha}$ is

$$
\mathcal{S}_{\alpha}=\sum_{\gamma} N S_{\gamma}(X),
$$

where the sum is over all weak compositions $\gamma$ that collapse to $\alpha$.

We shall see later that these functions are indeed quasisymmetric, but for now we focus on their connection to Schur functions. An example of a quasisymmetric Schur function is given in Figure 1. 

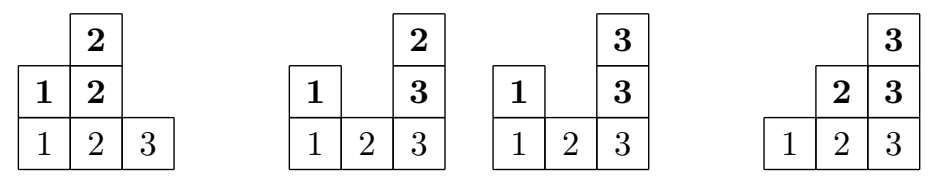

Fig. 1: $\mathcal{S}_{1,2}=N S_{(1,2,0)}+N S_{(1,0,2)}+N S_{(0,1,2)}=x_{1} x_{2}^{2}+x_{1} x_{2} x_{3}+x_{1} x_{3}^{2}+x_{2} x_{3}^{2}$

Recall that the Schur function $s_{\lambda}$ decomposes into the sum of $N S_{\gamma}$ over all compositions $\gamma$ such that $\lambda(\gamma)=\lambda$. This implies that the Schur function decomposes into a sum of quasisymmetric Schur functions

$$
s_{\lambda}=\sum_{\alpha: \lambda(\alpha)=\lambda} \mathcal{S}_{\alpha}
$$

Another observation we can make about these functions is that if $s_{\lambda}=\mathcal{S}_{\alpha}$ for a single composition $\alpha$, then the parts in the composition $\alpha$ must all be equal. In other words, if $\alpha=\left(\alpha_{1}, \alpha_{2}, \ldots, \alpha_{k}\right)$, then $\alpha_{1}=\alpha_{2}=\cdots=\alpha_{k}$.

\subsection{Connection to fundamental quasisymmetric functions}

The following proposition provides a description of the quasisymmetric Schur functions, $\mathcal{S}_{\alpha}$, as a sum of fundamental quasisymmetric functions. The proof requires the bijection $\Psi$ between SSYTx and SSAFs, and we refer the reader to (6) for further details.

Proposition 2.1 Let $\alpha$ be a composition of $n$. Then

$$
\mathcal{S}_{\alpha}=\sum_{T} F_{\beta(D(T))}
$$

where the sum is over all SYTX Tof shape $\lambda(\alpha)$ that map under $\Psi$ to a composition that collapses to $\alpha$.

Proof: Consider the bijection $\Psi$ between semi-standard Young tableaux and semi-skyline augmented fillings. Each SSYT is mapped to a unique SSAF under this map. The polynomial $F_{\beta(D(T))}$ consists of the weights of all SSYTx that standardize to $T$. But an SSYT $P$ that standardizes to $T$ maps to an SSAF that standardizes to $\Psi(T)$. The collapse of the standardization of an SSAF $P$ is equal to the collapse of $P$. This means that $\mathcal{S}_{\alpha}$ contains all SSAFs whose shape collapses to $\alpha$. Consider the collection of all SYTx that map to SAFs whose collapse is $\alpha$. The descent sets of these SYTx form the indices for the fundamental quasisymmetric functions that sum to $\mathcal{S}_{\alpha}$.

We will now show that, in fact, the set of all quasisymmetric Schur functions forms a basis for $\mathcal{Q}$. Before we do this, we will work towards and prove two lemmas.

For a composition $\alpha$, let $T_{\alpha}$ be the unique SYT of shape $\lambda(\alpha)$ and $\beta(D(T))=\alpha$. To see that $T_{\alpha}$ exists form the array $U_{\alpha}$

$$
\begin{array}{cccc}
1 & 2 & \cdots & \alpha_{1} \\
\alpha_{1}+1 & \cdots & \alpha_{1}+\alpha_{2} & \\
\vdots & & & \\
\cdots & n & &
\end{array}
$$


and then move every number as far north as possible. To see that $T_{\alpha}$ is unique, note that the number of descents in $T_{\alpha}$ is one less than the number of rows in $T_{\alpha}$ and so all entries in the first column except 1 must be all $i+1$ such that $i \in D\left(T_{\alpha}\right)$. This and the fact that $T_{\alpha}$ must be a SYT yield uniqueness.

Example 2.2 If $\alpha=(1,3,2)$ then $U_{\alpha}=$\begin{tabular}{ll|l|l|l}
1 & 2 & 3 & 4 \\
5 & 6
\end{tabular} and $T_{\alpha}=$\begin{tabular}{|l|l|l}
\hline 1 & 3 & 4 \\
\cline { 1 - 2 } 2 & 6 &
\end{tabular} .

Lemma 2.3 For a composition $\alpha=\left(\alpha_{1}, \alpha_{2}, \ldots, \alpha_{k}\right) \vDash n$, performing $\Psi$ on $T_{\alpha}$ yields the SSAF (basement included)

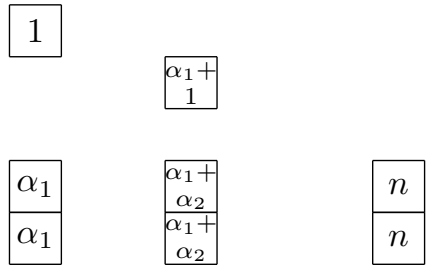

Proof: We proceed by induction on $n$. The result is clear for $n=1$. Now assume it is true for all compositions of $n-1$ and consider a composition $\alpha$ of $n$. We have two cases to consider:

1. $n$ is adjacent to $n-1$ in $U_{\alpha}$,

2. $n$ is not adjacent to $n-1$ in $U_{\alpha}$.

In the latter case $\alpha_{k}=1$ and thus $n$ will be the last symbol to be inserted so the SSAF (basement included) produced by induction will be

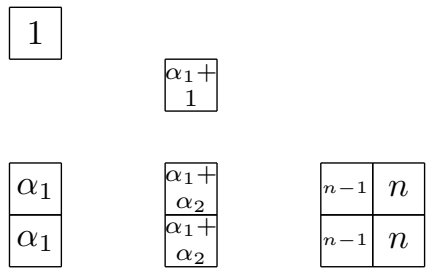

In the former case let $\tilde{T}_{\alpha}$ be the SYT of shape $\lambda(\tilde{\alpha})$ and $\beta\left(D\left(\tilde{T}_{\alpha}\right)\right)=\tilde{\alpha}$ where $\tilde{\alpha}=\left(\alpha_{1}, \alpha_{2}, \ldots, \alpha_{k}-1\right)$. The entries will be inserted in the same order as when performing $\Psi$ on $\tilde{T}_{\alpha}$ except at some stage $n$ will be inserted. This will lead to a nonzero height "building" in the $n$-th column standing on top of the basement cell filled with $n$. Consequently elements will be placed where they were when performing $\Psi$ on $\tilde{T}_{\alpha}$ or they will be placed in the $n$-th column, which will have a building of greater height than the $n$-1-th column of height zero. Thus, the elements will eventually be bumped to the column they occupied when performing $\Psi$ on $\tilde{T}_{\alpha}$ or if this was the $n$-1-th column they will now be found in the $n$-th column.

Lemma 2.4 $F_{\alpha}$ will always be a summand of $\mathcal{S}_{\alpha}$ with coefficient 1 .

Proof: This follows immediately from the existence and uniqueness of $T_{\alpha}$ and Lemma 2.3 
Let $>$ be the following order on compositions of $n$. If $\alpha$ and $\beta$ are compositions then $\alpha>\beta$ if and only if $\lambda(\alpha)$ is lexicographically greater than $\lambda(\beta)$ or if $\lambda(\alpha)=\lambda(\beta)$ and $\alpha$ is lexicographically greater than $\beta$. For example when $n=4$ we have

$$
(4) \triangleright(3,1) \triangleright(1,3) \triangleright(2,2) \triangleright(2,1,1) \triangleright(1,2,1) \triangleright(2,1,1) \triangleright(1,1,1,1)
$$

We are now ready to prove that quasisymmetric Schur functions form a basis for $\mathcal{Q}$.

Proposition 2.2 The set $\left\{\mathcal{S}_{\alpha} \mid \alpha \vDash n\right\}$ forms a $\mathbb{Z}$-basis for $\mathcal{Q}$.

Proof: For a fixed $n$ and $\alpha=\left(\alpha_{1}, \ldots, \alpha_{\ell(\alpha)}\right) \vDash n$ consider the summand $F_{\delta}$ appearing in $\mathcal{S}_{\alpha}$. By Proposition 2.1 we know that $\ell(\delta) \geq \ell(\alpha)$. If $\ell(\delta)=\ell(\alpha)$ then it follows that $\lambda(\alpha) \geq_{\text {lex }} \lambda(\delta)$ because if not then the first $i$ when $\lambda(\delta)_{i}>_{\text {lex }} \lambda(\alpha)_{i}$ will yield that row $i$ in any SYT of shape $\lambda(\alpha)$ is such that it cannot be filled without having, in total, more descents than $\ell(\alpha)-1$. If $\lambda(\alpha)=\lambda(\delta)$ then by Lemma 2.3 and the uniqueness of $T_{\alpha}$ we know the coefficient of $F_{\delta}$ will be 0 unless $\alpha=\delta$.

Let $M$ be the matrix whose rows and columns are indexed by $\alpha \vDash n$ ordered by and entry $M_{\alpha \delta}$ is the coefficient of $F_{\delta}$ in $\mathcal{S}_{\alpha}$. By the above arguement and Lemma 2.4 we have that $M$ is upper unitriangular, and the result follows.

The following proposition characterizes the quasisymmetric Schur functions that are obtained from only one fundamental quasisymmetric function.

Proposition $2.3 \mathcal{S}_{\alpha}=F_{\alpha}$ if and only if $\alpha=\left(m, 1^{e_{1}}, 2,1^{e_{2}}, \ldots, 2,1^{f}\right)$ where $m, f, e_{i}$ are nonnegative integers such that $m \neq 1, f \geq 0$, and $e_{i} \geq 1$ for all $i$.

Proof: To clarify exposition, in this proof when we refer to the height of a column in an SAF we refer to the number of cells above the basement.

To see that if $\alpha=\left(m, 1^{e_{1}}, 2,1^{e_{2}}, \ldots, 2,1^{f}\right)$ where $m, f, e_{i}$ are nonnegative integers such that $m \neq 1$, $f \geq 0$, and $e_{i} \geq 1$ for all $i$, then $\mathcal{S}_{\alpha}=F_{\alpha}$, consider a skyline augmented filling $F$ whose shape collapses to $\alpha$. If a column of height 2 immediately follows a column of height 1 , then any filling of the cells in these columns violates the type $B$ inversion triple condition. Therefore each column of height 2 must immediately follow an empty column.

The last $f$ height one columns must be right justified for $F$ to be an SAF, since otherwise there would be positive integers less than or equal to $n$ with no place in the filling. These two constraints imply that the leftmost column of height 1 must be the $(m+1)$-th column. Therefore, the column of height $m$ must appear in the $m$-th column, for otherwise the number $m$ would have no place in the filling $F$. Thus, the shape of the diagram is $\left(0^{m-1}, m, 1^{e_{1}}, 0,2,1^{e_{2}}, \ldots, 0,2,1^{f}\right)$ and a nonempty column in the $j^{\text {th }}$ column is filled with the number $j, j-1, \ldots, h$, where $j-h+1$ is the height of this column. This implies that there is only one SAF whose shape collapses to $\alpha$, and therefore $\mathcal{S}_{\alpha}=F_{\alpha}$.

To see that $\mathcal{S}_{\alpha}=F_{\alpha}$ implies that $\alpha$ is of the above form, recall that the super filling of a skyline shape consists of filling all cells in column $i$ with $i$. Form the SSAF, $T$, that is the super filling of shape $\left(0, \ldots, 0, \alpha_{1}, \alpha_{2}, \ldots, \alpha_{\ell(\alpha)}\right)$. Now if $\alpha \neq\left(m, 1^{e_{1}}, 2,1^{e_{2}}, \ldots\right)$ where $m, e_{i}$ are nonnegative integers such that $m \neq 1$ and $e_{i} \geq 1$ for all $i$ then at least one of the following three scenarios occur:

1. there exists a column $i$ of height $\alpha_{j}$ and column $i+1$ of height $\alpha_{j}+k$ for $k \geq 2$,

2. there exists a column $i$ of height $\alpha_{j}$ for $\alpha_{j} \geq 2$ and column $i+1$ of height $\alpha_{j}+1$, 
3. there exists a column $i$ of height $\alpha_{j}$ for $\alpha_{j} \geq 2$ and column $i+1$ of height $\alpha_{j} \geq \alpha_{k} \geq 2$.

In each case we now alter $T$ to form $T^{\prime}$, respectively.

1. We change the top entry of column $i+1$ to $i$.

2. We form the super filling of shape

$$
\left(0, \ldots, 0, \alpha_{1}, \alpha_{2}, \ldots, \alpha_{j-1}, 0, \alpha_{j}, \ldots, \alpha_{\ell(\alpha)}\right)
$$

and change the top entry of column $i$ to $i-1$ and the top entry of column $i+1$ to $i$.

3. We form the super filling of shape

$$
\left(0, \ldots, 0, \alpha_{1}, \alpha_{2}, \ldots, \alpha_{j-1}, 0, \alpha_{j}, \ldots, \alpha_{\ell(\alpha)}\right)
$$

and change the top entry of column $i+1$ to $i-1$.

In each case $T^{\prime}$ is a SSAF whose corresponding monomial is not a monomial contributing towards $F_{\alpha}$ and we are done.

\section{A Pieri rule for quasisymmetric Schur functions}

A natural question to ask about quasisymmetric Schur functions is how many properties of Schur functions extend to quasisymmetric Schur functions? In this regard, there are many avenues to pursue. We begin by showing that the quasisymmetric Schur functions exhibit a Pieri rule that natually generalizes the original Pieri rule for Schur functions.

More precisely, given two Ferrers diagrams, $\lambda, \mu$ the skew diagram $\lambda / \mu$ consists of those cells of $\lambda$ that remain once the cells of $\mu$ have been removed from the top left corner. We say a skew diagram is a row strip if no two cells lie in the same column, and a column strip if no two cells lie in the same row. We denote the number of cells in $\lambda / \mu$ by $|\lambda / \mu|$. The Pieri rule for Schur functions can now be stated.

Theorem 3.1 (Pieri rule for Schur functions) Let $\lambda$ be a partition. Then

$$
s_{(n)} s_{\lambda}=\sum_{\mu} s_{\mu}
$$

where the sum is taken over all partitions $\mu$ such that

1. $\delta=\lambda / \mu$ is a row strip,

2. $|\delta|=n$.

Also,

$$
s_{\left(1^{n}\right)} s_{\lambda}=\sum_{\mu} s_{\mu}
$$

where the sum is taken over all partitions $\mu$ such that

1. $\epsilon=\lambda / \mu$ is a column strip, 
2. $|\epsilon|=n$.

To extend the Pieri rule to quasisymmetric Schur functions we need the following definition.

Definition 3.2 Let $\alpha=\left(\alpha_{1}, \ldots, \alpha_{\ell}\right)$ be a composition whose largest part is $m$, and let $s \in[m]$. If there exists index $1 \leq i \leq \ell$ such that $s=\alpha_{i}$ and $s \neq \alpha_{j}$ for all $j>i$, then define

$$
\operatorname{rem}_{s}(\alpha)=\left(\alpha_{1}, \ldots, \alpha_{i-1},(s-1), \alpha_{i+1}, \ldots, \alpha_{\ell}\right),
$$

otherwise define rem $m_{s}(\alpha)$ to be the empty composition. Let $S=\left\{s_{1}<\cdots<s_{j}\right\}$. Then define

$$
\operatorname{row}_{S}(\alpha)=\operatorname{rem}_{s_{1}}\left(\ldots\left(\operatorname{rem}_{s_{j-1}}\left(\operatorname{rem}_{s_{j}}(\alpha)\right)\right) \ldots\right) .
$$

Similarly let $M=\left\{m_{1} \leq \cdots \leq m_{j}\right\}$. Then define

$$
\operatorname{col}_{M}(\alpha)=\operatorname{rem}_{m_{j}}\left(\ldots\left(\operatorname{rem}_{m_{2}}\left(\operatorname{rem}_{m_{1}}(\alpha)\right)\right) \ldots\right) .
$$

We collapse $\operatorname{row}_{S}(\alpha)$ or $\operatorname{col}_{M}(\alpha)$ to obtain a composition if needs be.

For any row strip $\delta$ we denote by $S(\delta)$ the set of columns its skew diagram occupies, and for any column strip $\epsilon$ we denote by $M(\epsilon)$ the set of rows its skew diagram occupies. We are now ready to state our extended Pieri rule.

Theorem 3.3 (Pieri rule for quasisymmetric Schur functions) Let $\alpha$ be a composition. Then

$$
\mathcal{S}_{(n)} \mathcal{S}_{\alpha}=\sum_{\beta} \mathcal{S}_{\beta}
$$

where the sum is taken over all compositions $\beta$ such that

1. $\delta=\lambda(\beta) / \lambda(\alpha)$ is a row strip,

2. $|\delta|=n$,

3. $\operatorname{row}_{S(\delta)}(\beta)=\alpha$.

Also,

$$
\mathcal{S}_{\left(1^{n}\right)} \mathcal{S}_{\alpha}=\sum_{\beta} \mathcal{S}_{\beta}
$$

where the sum is taken over all compositions $\beta$ such that

1. $\epsilon=\lambda(\beta) / \lambda(\alpha)$ is a column strip,

2. $|\epsilon|=n$,

3. $\operatorname{col}_{M(\epsilon)}(\beta)=\alpha$.

Rather than give the proof, which is similar in approach to that for Schur functions given in (1), we conclude this extended abstract with an example to illustrate Theorem 3.3 . 
Example 3.4 For a more visual interpretation of Theorem 3.3 we extend the definition of Ferrers diagram by saying a composition diagram of a composition $\alpha$ is an array of left justified cells with $\alpha_{i}$ cells in the $i$-th row from the top. Then rem $_{s}$ is the operation that removes the rightmost cell from the lowest row of length s.

For example, if we place $\bullet$ in the cell to be removed then

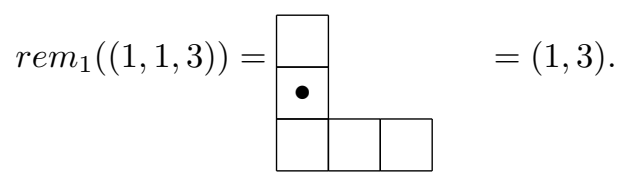

If we wish to compute $\mathcal{S}_{(1)} \mathcal{S}_{(1,3)}$ then we consider the skew diagrams

$$
(4,1) /(3,1),(3,2) /(3,1),(3,1,1) /(3,1),(3,1,1) /(3,1)
$$

with row strips containing one cell in column 4,2,1,1 respectively. Then

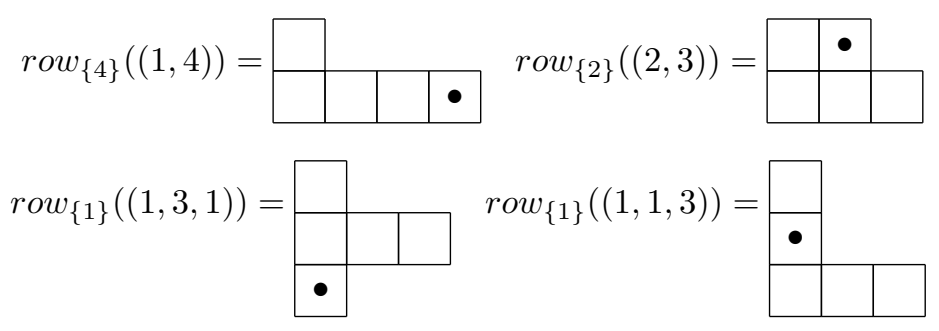

and hence

$$
\mathcal{S}_{(1)} \mathcal{S}_{(1,3)}=\mathcal{S}_{(1,4)}+\mathcal{S}_{(2,3)}+\mathcal{S}_{(1,3,1)}+\mathcal{S}_{(1,1,3)}
$$

\section{References}

[1] Fulton W., Young Tableaux, Cambridge University Press, Cambridge, UK, 1997.

[2] Gessel, I., Multipartite $P$-partitions and inner products of skew Schur functions, combinatorics and algebra (Boulder, Colo., 1983) 289-317, Contemp. Math., 34, Amer. Math. Soc., Providence, RI, 1984.

[3] Haglund, J., Haiman, M., and Loehr, N., A combinatorial formula for nonsymmetric Macdonald polynomials, Amer. J. of Math., to appear. arXiv.org/abs/math.CO/0601693

[4] Macdonald I., Symmetric Functions and Hall Polynomials, 2nd Edition, Oxford University Press, New York, USA, 1995.

[5] Macdonald, I., Affine Hecke algebras and orthogonal polynomials, Astérisque 237 (1996), 189-207, Séminaire Bourbaki 1994/95, Exp. no. 797.

[6] Mason, S., A decomposition of Schur functions and an analogue of the Robinson-Schensted-Knuth algorithm. arXiv:math.CO/0604430 